\title{
DETERMINANT COMPUTATIONS FOR SOME CLASSES OF TOEPLITZ-HANKEL MATRICES
}

\author{
ESTELLE L. BASOR AND TORSTEN EHRHARDT
}

\begin{abstract}
The purpose of this paper is to compute the asymptotics of determinants of finite sections of operators that are trace class perturbations of Toeplitz operators. For example, we consider the asymptotics in the case where the matrices are of the form $\left(a_{i-j} \pm a_{i+j+1-k}\right)_{i, j=0 \ldots N-1}$ with $k$ fixed. We will show that this example as well as some general classes of operators have expansions that are similar to those that appear in the Strong Szegö Limit Theorem. We also obtain exact identitities for some of the determinants that are analogous to the one derived independently by Geronimo and Case and by Borodin and Okounkov for finite Toeplitz matrices. These problems were motivated by certain statistical quantities that appear in random matrix theory.
\end{abstract}

Mathematics subject classification (2000): 47B35, 47B10, 82B. theory.

Keywords and phrases: Toeplitz operator, Hankel operator, determinant asymptotics, random matrix

\section{REFERENCES}

[1] J. BAIK, E. M. RAINS, Algebraic aspects of increasing subsequences, Duke Math. J. 109, no. 1 (2001), $1-65$.

[2] E. L. BASOR, T. EHRHARDT, Asymptotic formulas for determinants of a sum of finite Toeplitz and Hankel matrices, Math. Nachr. 228 (2001), 5-45.

[3] A. Borodin AND A. OKOUNKOV, A Fredholm determinant formula for Toeplitz determinants, Int. Eqns. Operator Th. 37 (2000) 386-396.

[4] A. BöTtCher AND B. Silbermann, Analysis of Toeplitz Operators, 2nd edition, Springer, Berlin 2006.

[5] T. EHRHARDT, A generalization of Pincusì formula and Toeplitz operator determinants, Arch. Math. (Basel), 80 (2003), 302-309.

[6] T. EHRHARDT, An new algebraic proof of the Szegö-Widom limit theorem, Acta Math. Hungar. 99, no. 3 (2003), 233-261.

[7] P. J. ForRester, N. E. FRANKEL, Applications and generalizations of Fisher-Hartwig asymptotics, J. Math. Phys. 45, no. 5 (2004), 2003-2028.

[8] V. V. PELLER, Hankel operators of the class $\mathscr{S}_{p}$ and their applications, Math. USSR Sbornik, 41, (1980/82), 443-479.

[9] V. V. PELLER, Hankel operators and their applications, Springer monographs in Mathematics, Springer, New York 2003.

[10] W. Rudin, Functional Analysis, McGraw-Hill, Inc., New York 1991.

[11] I. C. GOHBERG AND M. G. KREIN, Introduction to the Theory of Linear Nonselfadjoint Operators, Transl. Math. Monographs, Vol. 18, Amer. Math. Soc., Providence, 1969.

[12] M. L. MehtA, Random Matrices, Academic Press, San Diego, 1991.

[13] C. A. TRacy, H. Widom, Introduction to random matrices, in: Lecture Notes in Physics, Vol. 424, Springer, Berlin 1993, pp. 103-130.

[14] H. WIDOM, Block Toeplitz matrices and Determinants, Adv. in Math. 13, no. 3 (1974). 